\title{
Implicit motor sequence learning is not purely perceptual
}

\author{
DANIEL B. WILLINGHAM \\ University of Virginia, Charlottesville, Virginia
}

\begin{abstract}
Many reports have indicated that implicit learning of sequences in a choice response time task is primarily perceptual; subjects learn the sequence of stimuli rather than the sequence of motor responses. Three experiments tested whether implicit motor sequence learning could be purely perceptual; no support was found for that hypothesis. Subjects who merely watched stimuli did not learn the sequence implicitly (Experiment 1), and sequence learning transferred robustly to a different set of stimulus cues (Experiment 2). In the final experiment, the stimulus-response mapping was changed at transfer so that one group of subjects pushed the same sequence of keys but saw new stimuli, whereas another group pushed a different sequence of keys but saw the same stimuli. Transfer to the new mapping was shown only if the motor sequence was kept constant, not the perceptual sequence. It is proposed that subjects learn a sequence of response locations in this and similar tasks.
\end{abstract}

There has recently been some interest in perceptualmotor learning of sequences. This interest was generated by the implicit nature of the learning; subjects can learn perceptual-motor sequences, as revealed by performance measures, although they perform at chance on recognition or recall tests of the sequence (Willingham, Greeley, \& Bardone, 1993; Willingham, Nissen, \& Bullemer, 1989). Furthermore, amnesic patients who show profound impairment on such explicit tests as recall and recognition learn perceptual-motor sequences at a normal rate as adduced from performance measures (see, e.g., Nissen, Willingham, \& Hartman, 1989; Reber \& Squire, 1994).

It is therefore of interest to consider exactly what subjects learn when they learn implicit sequential information. Different task paradigms have been used, but many of these studies have used the serial response time (SRT) task. The SRT task is a four-choice RT task in which the stimuli appear in a repeating sequence (10 or 12 units long), although the subjects are not told about the sequence. Normal subjects are said to show implicit knowledge of the sequence if their RTs decrease when the sequence is present but increase again when the stimuli appear randomly. Subjects may also gain explicit knowledge of the sequence. This knowledge is usually assessed by using a recognition or recall test administered after the RT task.

There are several ways that subjects might learn a sequence implicitly. They may learn the sequence of percep-

The assistance of Sarah Driscoll, Tim O'Hara, Eve Peterson, Stephanie Preston, and R. Cannon Thomas is gratefully acknowledged. The author thanks Alice Healy and Paul Reber for helpful comments on an earlier version of this article. Correspondence concerning this article should be addressed to D. B. Willingham, Department of Psychology, University of Virginia, 102 Gilmer Hall, Charlottesville, VA 22903 (e-mail: willingham@virginia.edu). tual stimuli, which would essentially be a training of attention; the subjects are already attending to the correct spatial location when the stimulus appears and so can respond to it more quickly. Subjects may learn a sequence of motor responses - that is, a motor program. They may learn a sequence of locations in egocentric space which serve as targets for response. A final possibility is that they may learn a sequence of response selections. A response selection is a hypothetical stage of information processing in which a subject chooses an action based on a perceptual cue (Welford, 1976). Evidence relevant to these hypotheses will be reviewed in turn.

\section{Evidence That Learning is Not Motoric}

There is good evidence that learning in the SRT task is not purely motoric, at least not in the sense of being specific to an effector. A. Cohen, Ivry, and Keele (1990) trained subjects on the SRT task and then transferred them to a version in which they responded with one finger, like a "hunt and peck" typist. They reported excellent transfer and concluded that learning was not specific to the motor output used at training. In a still more complete set of experiments examining the effect of transferring knowledge to a new effector, Keele et al. (1995) reached the same conclusion.

\section{Evidence That Learning is Perceptual}

Consistent with the idea that learning is not motoric, other experiments have indicated that learning might be purely perceptually based. Stadler (1989) reported perceptually based learning. He used a visual search task in which the screen quadrant in which the target appeared was predictable from the previous six trials, based on a complex rule. There were many such rules, but, after considerable training, subjects showed reliable knowledge of the rules, as revealed by increased RTs on the critical seventh trial when the rules were not followed. Stadler then 
had subjects use different fingers to respond, or he changed the location of the target on the critical trials; it appeared in the correct quadrant, but in the corner opposite to the corner in which it had appeared. Changing the motor response had a negligible impact on RTs. Changing the positions had a marginal $(.05<p<.10)$ effect, but the small number of subjects $(N=4)$ did not afford much power.

Howard, Mutter, and Howard (1992) reported that sequence learning in the SRT task was purely perceptual. They had subjects either perform the SRT task or simply watch the stimuli without responding. On the test blocks, all subjects responded, and the subjects who had simply watched showed evidence of learning equal to that of the subjects who had responded during training. A cued recall test revealed that explicit knowledge was very high for the subjects who had watched the stimuli, however; they reproduced the sequence with an average $95 \%$ accuracy. It is possible that the performance advantage was conferred by explicit knowledge (cf. Curran \& Keele, 1993).

Fendrich, Healy, and Bourne (1991, Experiment 2) used a data-entry task. At training, subjects entered a number of digit sequences on a keypad. The key configuration was changed at test. The necessary motor sequence was therefore changed for repeated digit sequences. Other sequences were changed in such a way that the motor sequence matched the one used at training, and still other digit sequences were completely novel. Novel sequences were entered more slowly than those that matched training sequences perceptually or motorically, and the authors concluded that implicit knowledge may be represented both motorically and perceptually. The interpretation of these data is complicated, however, by a strong dependence between recognition scores of the sequences taken during the second week and the RT advantage. It is possible that the advantage was conferred largely by explicit memory, as in the Howard et al. (1992) study.

Mayr (1996) dissociated the location of stimuli and the necessary response by having stimuli appear in different locations, but instructing subjects to respond to the shape of the stimulus. The subjects experienced uncorrelated sequences of spatial locations and sequences of movements. Like Fendrich et al. (1991), Mayr reported parallel learning of both spatial and motor sequences.

\section{Evidence That Learning Is Not Perceptual}

Willingham et al. (1989) concluded that the learning was neither purely perceptual nor purely motoric. In a paradigm similar to Mayr's (1996), they dissociated perceptual and motor information by using colored stimuli. The stimuli appeared in one of four spatial positions, but subjects responded to color. Thus, the subjects saw a repeating sequence of stimulus positions (but pushed random buttons) or pushed a sequence of buttons (but saw random positions). Subjects learned in the latter condition but not in the former. When the subjects were transferred to the typical SRT task (in which cues are spatial), however, there was no transfer of the learning. Willing- ham et al. (1989) concluded that learning was neither perceptual, nor encapsulated in the motor system. They concluded that learning might be based on some information-processing stage (such as response selection) that occurs between perception and action.

Zießler (1994) used a variant of the SRT task that also entailed visual search, and varied the response requirements; some subjects used a distinct motor response for each stimulus, whereas others responded to many stimuli with the same response. Zießler reported much better learning in the former condition than in the latter and concluded that this indicated that learning in serial pattern tasks was not purely perceptual, that the motor responses were crucial to learning.

\section{Summary of Implicit Sequence Learning Studies}

Although different methodologies were used in these studies, implicit sequence learning was examined in all of them. They can be summarized thus: Implicit sequence learning is not strictly motoric in the sense that knowledge is specific to an effector (A. Cohen et al., 1990; Keele et al., 1995). Most researchers argue that purely perceptual information can support learning (Fendrich et al,, 1991; Howard et al., 1992; Mayr, 1996; Stadler, 1989).

Closer investigation, however, shows that the evidence may not be that compelling. In two of the studies (Fendrich et al., 1991; Howard et al., 1992), subjects may have acquired significant explicit knowledge. In the other, the effects were marginal (Stadler, 1989). Furthermore, as Stadler points out, what appears to be a perceptual task may involve eye movements, possibly allowing for ocular motor learning, which may be relevant to Mayr's (1996) findings.

Two studies have reported that perceptual learning is poor or fragmentary (Willingham et al., 1989; Zießler, 1994), but both of these studies have a limitation; one cannot be certain that subjects attended to the aspect of the stimuli that was the basis of the sequence. In Zießler's (1994) study, subjects saw a spatial sequence of stimuli but were asked to press a single key in response to most of the stimuli-one stimulus was a "catch" stimulus that required a different response. It may be that the subjects differentiated the stimuli into only two categories, corresponding to the two response choices, meaning that they attended to each stimulus only insofar as was necessary to make this categorization judgment. Under normal circumstances, however, each stimulus is paired with a unique response, requiring attention to more differentiating characteristics of the stimuli. In Willingham et al.'s (1989) study, the subjects did not respond to spatial position and therefore may not have attended to it, and the perceptual sequence was one of spatial positions. It may have been necessary to attend to spatial location in Mayr's (1996) experiment because the positions were much farther apart.

Thus, although there has been considerable effort to elucidate the representation of implicit sequence learning, there are conflicting data, and the extant experiments ap- 
pear to be open to alternative explanations. The purpose of the work reported here was to test the hypothesis that implicit sequence learning could be supported by purely perceptual information, while ensuring that apparent perceptually based learning was not due to explicit knowledge, or to eye movements, and also ensuring that subjects attended to the relevant perceptual cues.

\section{EXPERIMENT 1}

Howard et al. (1992) offered what is arguably the conceptually clearest test of the hypothesis that implicit sequence learning can be supported by purely perceptual information. They asked subjects simply to watch stimuli, and then used a performance measure to test whether they had implicitly learned the sequence. A problem in that study was that the subjects gained significant explicit knowledge, making it unclear whether the RT advantage they showed was due to implicit or explicit learning. Experiment 1 was designed to replicate Howard et al.'s experiment, but to reduce the level of explicit knowledge that the subjects acquired.

In this experiment, the stimuli appeared randomly in the first trial block. In prior research, postexperimental interviews had shown that many subjects become aware of the sequence because they suspect that they are not told all the details of the experimental procedure; that is, they suspect that the stimuli are sequenced. Once they search for the sequence, they find it. It was hoped that these subjects would be discouraged from continuing to search for the sequence after failing to find one in the first, random block. A large number of subjects were tested, so that any who had gained significant explicit knowledge could be eliminated from the analysis.

\section{Method}

Subjects. Eighty-two undergraduates (38 male) at the University of Virginia participated in return for extra credit in an introductory psychology course. They were randomly assigned to conditions as they entered the experiment without the constraint that equal numbers of subjects te assignied to cach condition.

Stimuli and Apparatus. Stimuli were generated on a video monitor controlled by a Macintosh IIci microcomputer. Four boxes, corresponding to the four possible stimulus locations, appeared continuously on the screen. The boxes were $2 \mathrm{~cm}$ square, and the center-to-center distance was $5 \mathrm{~cm}$. They were arranged horizontally and centered vertically. The stimulus on an individual trial was an asterisk $(0.3 \mathrm{~cm}$ in diameter $)$ that appeared at the center of one of the boxes.

The subjects responded to the stimuli using the " $\mathrm{Z}$," "C," " $\mathrm{B}$," and "M" keys, with the leftmost key corresponding to the leftmost stimulus, and so on. The keys were covered with white paper.

Procedure. In the push condition, the subjects $(N=24)$ were told to rest the index and middle fingers of each hand on the four response keys. An asterisk appeared in one of the four stimulus positions, and the subject pushed the response key directly below the stimulus. If the subject pushed the wrong key, a tone sounded for $120 \mathrm{msec}$, and the subject continued to respond until the correct key was pressed. When the subject responded correctly, the stimulus disappeared, there was a $250-\mathrm{msec}$ pause, and then a new stimulus appeared.
Other subjects were not required to press keys. They simply watched the stimuli. Each stimulus appeared for $300 \mathrm{msec}$, whereupon it was erased; this was followed by a $250-\mathrm{msec}$ pause before a new stimulus appeared. Subjects in the watch random $(N=28)$ condition saw randomly appearing stimuli. Subjects in the watch sequence $(N=28)$ condition saw stimuli that appeared in a repeating sequence.

All subjects saw 96 stimuli in each trial block. In the training phase, there were four trial blocks, with a 45 -sec rest break between blocks. For the watch sequence and push groups, the first training block showed random stimuli and the remaining three showed sequenced stimuli. The sequences were selected (and the random blocks were created) from a corpus of 563 twelve-unit sequences. The sequences met the following constraints: the same stimulus could not appear on successive trials; each stimulus appeared an equal number of times; the sequence could not contain a run of four stimuli (e.g., 4-3-2-1) or a trill of four (e.g., 1-2-1-2).

After the training phase, all subjects participated in a transfer phase, during which they were asked to respond to the stimuli. The subjects responded to two random-trial blocks, a sequenced block, and a final random block. The initial two random-trial blocks were administered to give subjects who had been watching stimuli during training a chance to adjust to the new task requirements, for example, to learn the stimulus-response mapping and the responseto-stimulus interval. There was a brief break after the training phase, during which the subjects received new instructions for the transfer phase.

At the end of the transfer phase, the subjects were told that the stimuli had appeared in a repeating sequence and then were asked to reproduce the sequence on the keyboard in a free recall task. Their responses were echoed on the screen. They were told to push the "Q" button when they had produced as much of the sequence as they could remember. A maximum of 26 responses was allowed.

\section{Results and Discussion}

Explicit knowledge. The free recall test was scored by taking the sum of all correct responses, whether they were consecutive or not, but a response had to be part of a segment of at least three correct positions. For example, if a subject saw the sequence 243234121431 and then produced " 14 " on the free recall test, the score would be 0 , the rationale being that " 14 " occurs by chance in too many sequences for this response to signify explicit knowledge. Suppose the subject produced " 412243 " on the free recall test. The score would be 6 , because " 412 " and " 243 " appear in the sequence. If the subject produced " 2431 ," the score would be 4 ; although " 243 " and " 431 " both appear the sequence, the segments overlap, and the " 43 " the subject recalled counts only once.

The free recall scores appear in Table 1 . An analysis of variance (ANOVA) shows a reliable difference among conditions $\left[F(2,78)=4.7, p<.05, M S_{\mathrm{e}}=6.3\right]$, and followup contrasts show that the watch sequence condition had reliably higher free recall scores than did the watch random condition $\left[F(1,78)=9.5, p<.005, M S_{\mathrm{e}}=6.3\right]$. No other differences were reliable $(p s>.10)$. Thus, subjects in the watch sequence condition, on average, acquired some explicit knowledge of the sequence.

Response times. Accuracy tends to be very high in the SRT task because subjects are told to respond as quickly as possible without making errors. The subjects in all of the experiments averaged $90 \%$ or better across trial blocks, 


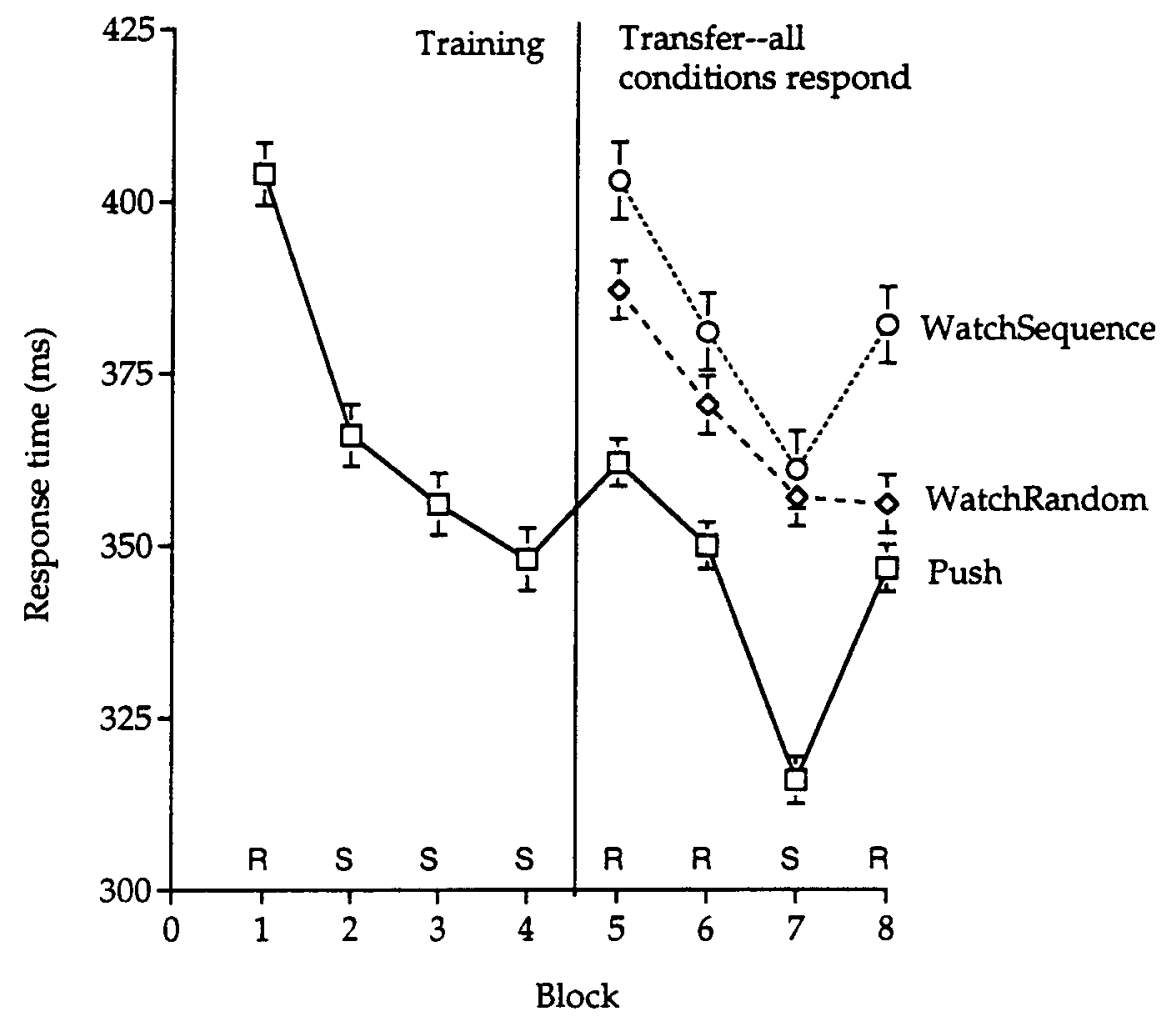

Figure 1. Response times across training and transfer, separated by condition, for Experiment 1 . Error bars are standard errors, calculated within subjects. $R$, random stimuli; $S$, sequenced stimuli.

and accuracy differences were never reliable. Accuracy will not be further discussed.

RT is taken to be the primary dependent measure. RTs were summarized by dividing each block into eight sets of 12 trials. The median of each set was calculated, and then the mean of the eight medians was calculated, which served as the summary RT for the trial block. As shown in Figure 1, subjects who responded to stimuli during the training phase reliably improved their RTs during training $\left[F(3,69)=30.7, p<.001, M S_{\mathrm{e}}=475\right]$.

The crucial RT data are those obtained at transfer, shown in Figure 1. There was a reliable effect of trial block $\left[F(3,231)=36.2, p<.001, M S_{\mathrm{e}}=584\right]$, reflecting the fact that the subjects showed much faster RTs on the sequenced trial block than on the random blocks. There was also a reliable main effect of condition; subjects who had responded during training responded more quickly than did subjects who had not $\left[F(2,77)=3.8, p<.05, M S_{\mathrm{e}}=\right.$ $10,090]$. The difference in the random and sequence blocks varied by condition, however $[F(6,231)=2.3, p<.05$, $M S_{\mathrm{e}}=585$ ]. To address the question of learning in a more focused way, a learning measure was calculated for each subject - the difference between RTs during the sequenced block and the mean RT during the adjacent random blocks. This measure showed a reliable effect of condition $\left[F(2,77)=4.4, p<.05, M S_{\mathrm{e}}=1,043\right]$ due to very poor transfer by the watch random condition $(M=6.2$;
$S E=5.1)$ compared with the watch sequence and push conditions $(M \mathrm{~s}=20.2$ and 32.6, respectively; $S E \mathrm{~s}=7.8$ and 5.3). These data are shown in the left part of Figure 2.

The watch sequence learning score is not reliably different from the push learning score $[F(1,77)=1.8, p=.17$, $\left.M S_{\mathrm{e}}=1,043\right]$. It is marginally different from the score of the watch random condition $\left[F(1,77)=2.7, p=.11, M S_{\mathrm{e}}=\right.$ $1,043]$. The watch sequence learning score is reliably different from zero $[t(27)=2.6, p<.05]$. The comparison with the watch random condition is the more decisive, because subjects in the watch sequence condition might show faster RTs to the sequence at transfer not because they learned the sequence during training, but because they learned it within the single block of training during the transfer task. The comparison with the watch random condition controls for this possibility.

Thus, the pattern of data appears to partially replicate Howard et al. (1992); subjects who merely watched the stimuli show statistically marginal evidence of having learned the sequence. The picture is quite different when one examines these data in light of the subjects' performance on explicit tests, however. Post hoc evaluation of the free recall data showed that a small number of subjects showed very high levels of explicit knowledge and also very high levels of learning at transfer. The transfer data were reanalyzed, removing from the analysis those subjects who successfully produced 10 or more positions 


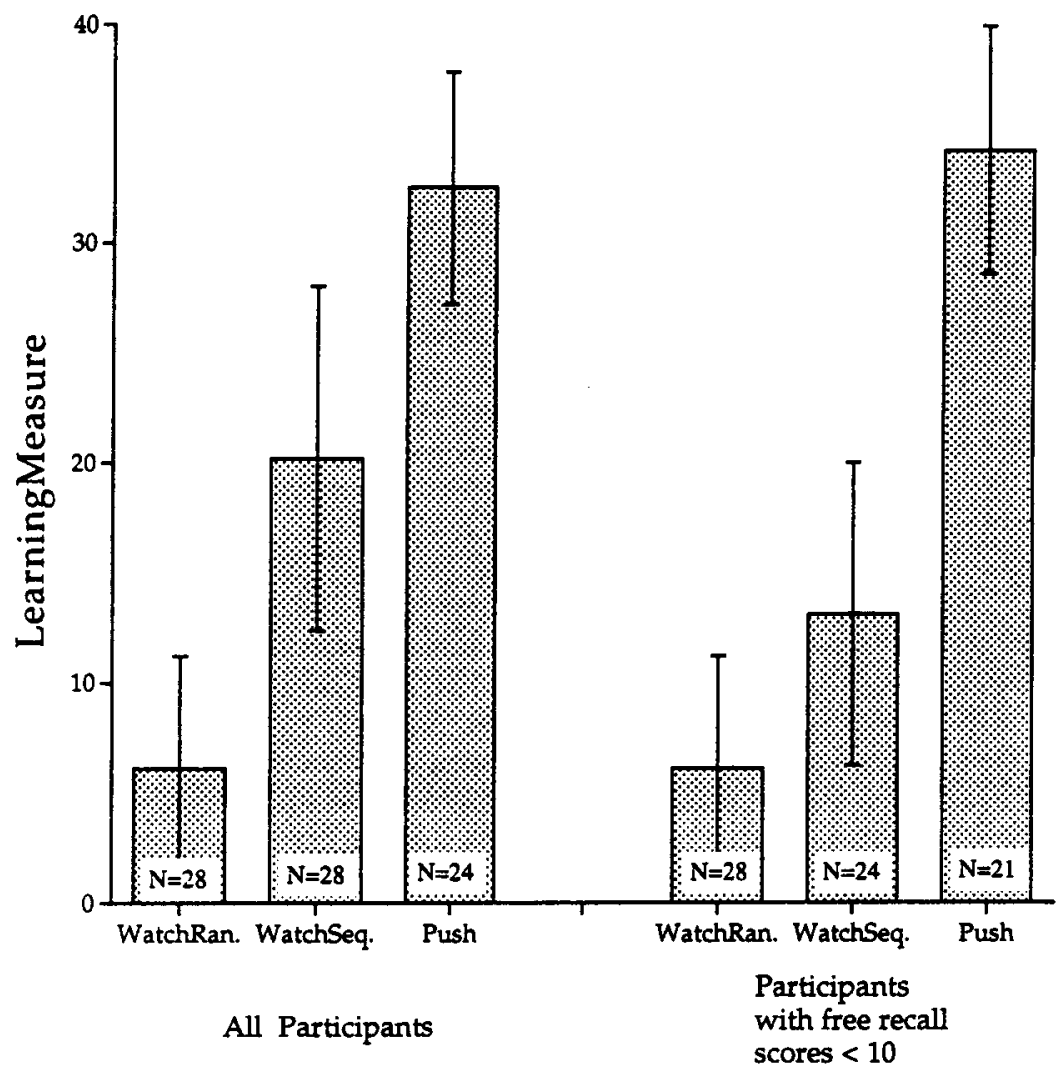

Figure 2. Summary learning measure at transfer for Experiment 1, comparing results for all subjects (at left) and when subjects with free recall scores of 10 or higher are removed (at right). Error bars are standard errors.

of the sequence at free recall (watch random, $n=0$; watch sequence, $n=4$; push, $n=3$ ). The overall ANOVA qualitatively replicated the results when all of the subjects were included in the analysis, showing that there was a difference among the conditions in the pattern of RTs across blocks, but the learning was actually quite different, as shown in the right part of Figure 2.

The learning measure at transfer was reanalyzed, and again showed a difference among the conditions $[F(2,70)=$ $\left.5.9, p<.01, M S_{\mathrm{e}}=828\right]$. Planned contrasts showed that the push condition showed greater transfer than the watch sequence condition $\left[F(1,70)=6.0, p<.05, M S_{\mathrm{e}}=828\right]$, which showed transfer equivalent to that of the watch random condition $\left[F(1,70)=0.75, p>.20, M S_{\mathrm{e}}=828\right]$. The means for the conditions were $34.2,13.1$, and $6.2 \mathrm{msec}$, respectively. Again, the watch sequence condition's learning score was reliably above zero, but zero is not the appropriate baseline of comparison because learning occurs during the single block of training that subjects receive at transfer. The appropriate comparison for the watch sequence condition is the watch random condition, and they are not reliably different.

Still, one cannot conclude from this study that perceptual learning does not occur at all. It is possible that perceptual experience makes some contribution to learning, but that this experiment afforded insufficient power to detect it; naturally, quite a lot of power is required to conclude that an effect is absent, if one allows a small effect to be of interest. The present experiment does allow for the conclusion that perceptual information alone cannot support implicit sequence learning.

Experiment 1 replicated Howard et al. (1992) in showing that subjects who simply watched the stimuli showed faster RTs in response to the sequence in a transfer task, and this advantage was not reliably different from that shown by subjects who had responded motorically to the stimuli during training; still, the statistical difference compared with a condition in which subjects watched random stimuli was marginal. By removing from all conditions the few subjects with very complete explicit knowledge of the sequence, the watch sequence condition's learning is made indistinguishable from that of the watch random condition. Thus, perceptual experience is not a substitute for motor practice.

One difficulty with this study, however, is the extent to which one can be certain that subjects were indeed watching the stimuli, although they report that they were. To be certain of this, one could measure eye movements, but then one might wonder whether what is purportedly perceptual learning is in fact the learning of a sequence of 
Table 1

Free Recall, Experiment 1

\begin{tabular}{lcc}
\hline \multicolumn{1}{c}{ Condition } & $M$ & SEM \\
\hline Push & 4.2 & 0.4 \\
Watch random & 3.1 & 0.4 \\
Watch sequence & 5.1 & 0.6 \\
\hline
\end{tabular}

eye movements. Experiment 2 addressed these problems by having subjects respond to stimuli during training, thus ensuring that they were attending to them. The stimuli in the critical experimental condition were centrally presented digits, so a sequence of eye movements could not be learned. Subjects were first trained to respond either to digits or to spatial stimuli. Then all subjects were transferred to spatial stimuli. If purely perceptual information supports implicit sequence knowledge, or if knowledge is based on stimulus-response pairings, the knowledge should not transfer to a new task in which the stimuli are changed.

\section{EXPERIMENT 2}

\begin{abstract}
Method
Subjects. Fifty-two undergraduates (20 male) at the University of Virginia participated in return for extra credit in an introductory psychology course. The subjects were randomly assigned to conditions as they entered the experiment without the constraint that equal numbers of subjects be assigned to each condition.

Stimuli and Apparatus. The apparatus and spatial stimuli were identical to those of Experiment 1. For the digit stimuli, one box ( $2 \mathrm{~cm}$ square) was continuously visible at the center of the monitor screen and digits were presented within the box. They appeared in the New York font, and each digit was approximately $1.5 \mathrm{~cm}$ high and $1 \mathrm{~cm}$ wide. Responses were made as in Experiment 1; for the digit stimuli, the digits " 1 " through " 4 " were mapped on the four keys from left to right.

Procedure. The procedure was identical to that of Experiment 1, except that the subjects saw a fifth training block of random stimuli. Comparing this final random block with the fourth sequence block could be taken as a measure of sequence learning during the training phase. The experimental conditions were: the spatial condition $(n=18)$, in which sequenced spatial stimuli were seen during training; the sequence digits condition $(n=15)$, in which sequenced digits were seen during training; and the random digits condition $(n=19)$, in which only random digits were seen during training. At transfer, subjects in all conditions saw spatial stimuli. As in Experiment 1, four boxes were arranged horizontally, with an asterisk appearing in one of them to signify the stimulus for response. In the random digits condition, the subjects did not perform the free recall test of the sequence because they never saw the sequence, and the results of Experiment 1 had shown that the successful guessing rate was low in this task.
\end{abstract}

\section{Results and Discussion}

Explicit knowledge. The free recall data were analyzed as in Experiment 1 and are shown in Table 2. The sequence digits condition showed higher levels of explicit knowledge than did the spatial condition $[F(1,30)=5.0$, $\left.p<.05, M S_{\mathrm{e}}=6.4\right]$. The absolute levels of recall are not high, but given that chance performance is about three positions correct, as shown in Experiment 1, clearly some of the subjects gained some explicit knowledge.

Response times. RTs were summarized as in Experiment 1 . During training, there was a reliable effect of condition due to faster RTs in the spatial condition $[F(2,49)=$ $\left.22.4, p<.01, M S_{\mathrm{e}}=15,590\right]$. There was also a reliable main effect of block $\left[F(4,196)=32.2, p<.01, M S_{\mathrm{e}}=\right.$ $1,022]$, but that must be interpreted in light of the reliable interaction of condition and block $[F(8,196)=6.0, p<$ $\left..01, M S_{\mathrm{e}}=1,022\right]$. As is clear from Figure 3, the conditions with sequenced stimuli show an RT pattern indicative of sequence knowledge. They improve RTs across Blocks $1-4$ and show a large RT increase when the random stimuli are presented in Block 5; the random digits condition does not show this RT pattern.

The crucial question is whether the sequence knowledge would transfer from one type of stimulus to another, and the answer is that it does. As shown in Figure 3, both the spatial and the sequence digits conditions show a significant drop in RTs on the third transfer block when the stimuli are sequenced, but the random digits condition does not $\left[F(6,147)=2.9, p<.01, M S_{\mathrm{e}}=778\right]$.

It is possible that the transfer from the digit to the spatial stimuli is mediated through explicit memory. To evaluate that possibility, all subjects who correctly recalled 5 or more units of the sequence were eliminated from the analysis. Note that a criterion different from that in Experiment 1 is used; in Experiment 1 a lax criterion of explicit knowledge was used to show the fragility of the apparent implicit knowledge of the subjects who had only watched stimuli. In this experiment, a more stringent criterion was used to show the robustness of the transfer. The pattern of data at training was the same with this subset of subjects. The transfer data also retain the same pattern. Reliable sequence knowledge during the transfer task was shown by both the spatial $(N=15$; mean difference score $=32.8 \mathrm{msec}$ ) and the sequence digits conditions $(N=6$; mean difference score $=44.6 \mathrm{msec})$. The level of knowledge is not reliably different $[F(1,19)=0.5, p>.20$, $\left.M S_{\mathrm{e}}=974.9\right]$. To be certain that this finding was not an artifact of the criterion selected, a number of free recall criteria were selected (from 4 correct through 10 correct), and the results were qualitatively the same under all criteria.

These results are consistent with Experiment 1 in indicating that sequence learning in the SRT task is not solely perceptual. If it were, changing the stimuli should have reduced or eliminated the expression of implicit sequence knowledge, but the transfer manipulation had no effect. This experiment also indicates that learning is not based on stimulus-response pairings.

Experiment 2 ensured that subjects attended to perceptual cues by requiring that they respond to them, and then changed the perceptual information at transfer to see whether that change disrupted the expression of implicit learning. Experiment 3 also ensured that subjects attended to the stimuli during training and included transfer con- 


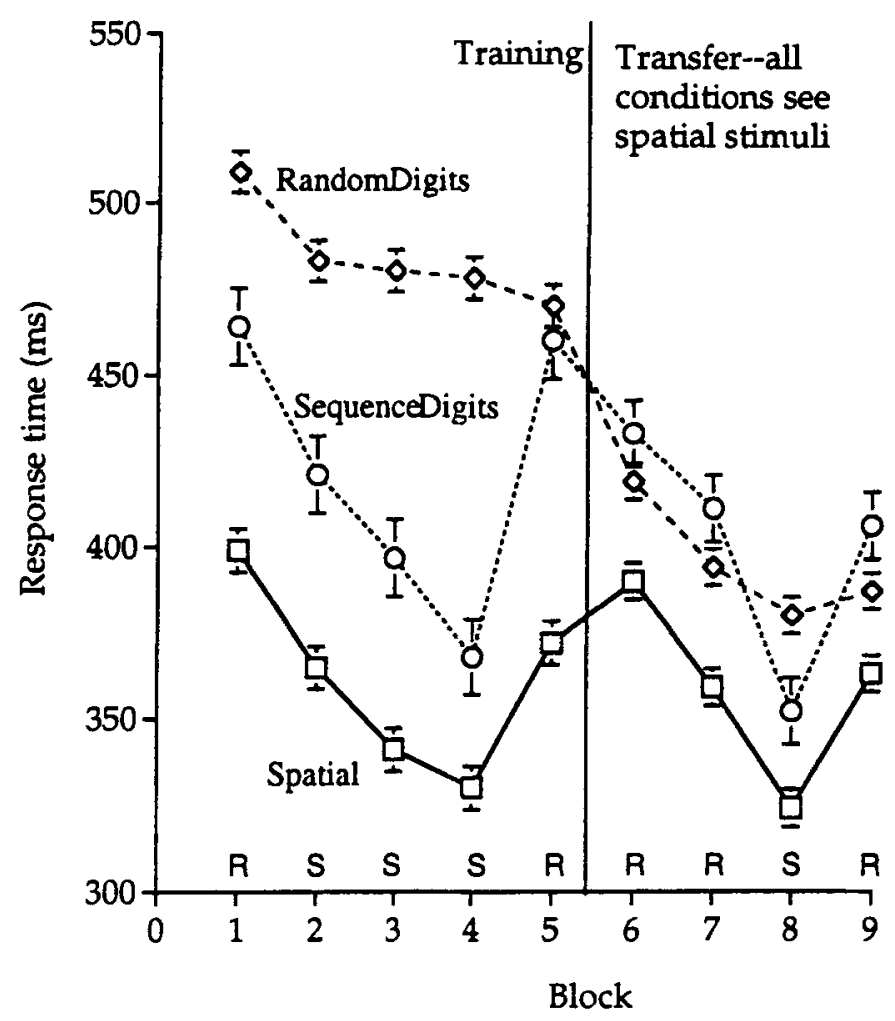

Figure 3. Response times across training and transfer, separated by condition, for Experiment 2. Error bars are standard errors calculated within subjects. $R$, random stimuli; $S$, sequenced stimuli.

ditions where either perceptual or motoric information alone might support sequence knowledge. The question was whether either or both conditions would show evidence of transfer.

\section{EXPERIMENT 3}

\section{Method}

Subjects. Seventy-three undergraduates $(24$ male) at the University of Virginia participated in return for extra credit in an introductory psychology course. The subjects were randomly assigned to one of three experimental conditions as they entered the experiment without the constraint that equal numbers of subjects be assigned to each condition. Two of the conditions (perceptual and motor) were of greater interest, so the assignment procedure was designed so that the chances of being assigned to one of these conditions was $50 \%$ greater than the odds of being assigned to a third, control condition (SRT).

Stimuli and Apparatus. Stimuli and apparatus were the same as those used in Experiment 1.

Procedure. With three exceptions, the procedure matched that of Experiment 2. First, centered digits were not used as stimuli in any of the conditions; only spatial stimuli were used. Second, in the perceptual $(N=29)$ and motor $(N=27)$ conditions, the subjects were trained on the SRT task using an incompatible stimulus-response mapping. Instead of responding by pressing the key directly below the stimulus, subjects pressed the key one position to the right of the stimulus. If the stimulus on the far right appeared, they were to "wrap around" and press the key on the far left. In the SRT $(N=17)$ condition, subjects performed the task with the normal, compatible mapping.

At transfer, all subjects used the compatible mapping, and all experienced the same sequence used during training; the sequence was either perceptually the same or motorically the same. In the perceptual condition, the stimuli were the same, but because the mapping had been changed, the subjects would press keys that were different from those they had pressed in training. In the motor condition, the stimulus sequence changed so that the subjects would press the same sequence of keys that they had during training, although they would, of course, be seeing a different sequence of stimuli.

The third difference in procedure from that of Experiment 2 was that the transfer consisted of only three blocks of trials. There was only one block of random stimuli before the sequenced stimuli were reintroduced. This change was made because it was thought that one block of trials might be sufficient for subjects to adjust to the new mapping and would lessen the chance of retroactive interference.

During the free recall test, subjects in the motor or perceptual condition were told that they could recall the sequence as they had seen it during the training session or during the transfer session, as they wished.

Table 2

Free Recall, Experiment 2

\begin{tabular}{lcc}
\hline Condition & $M$ & $S E M$ \\
\hline Spatial & 2.8 & 0.4 \\
Sequence digits & 4.8 & 0.8 \\
\hline
\end{tabular}




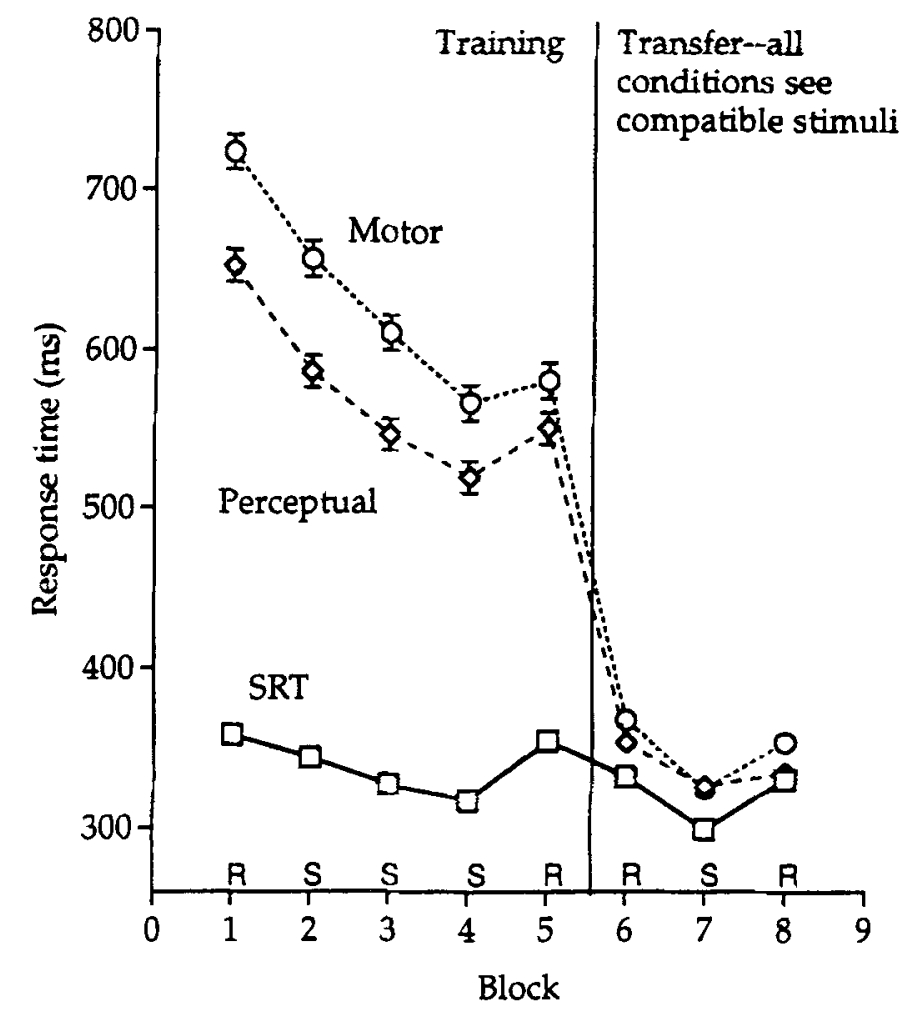

Figure 4. Response times across training and transfer, separated by condition, for Experiment 3. Error bars are standard errors calculated within subjects. Where error bars do not appear, they are too narrow to be printed. $R$, random stimuli; $S$, sequenced stimuli.

\section{Results}

Explicit knowledge. Explicit knowledge was unusually poor in this experiment. In the SRT condition, only 6 of the 17 subjects successfully recalled any of the sequence, and 5 of them recalled four or fewer positions; 1 subject correctly recalled six positions. There is not a ready explanation for this unusually poor free recall performance. None of the subjects in the motor or perceptual condition successfully recalled any positions. This result is not surprising, considering that the incompatible mapping was quite demanding of attention. Most of the subjects in the motor or perceptual condition were unwilling to attempt recall at all.

Response times. RTs were summarized as in Experiment 1 . The training data appear in Figure 4. There is a reliable effect of condition $[F(2,70)=31.2, p<.001$, $\left.M S_{\mathrm{e}}=73,206\right]$ because the SRT condition responded more quickly than the motor or perceptual condition, doubtless because they used a compatible mapping. There was also a reliable effect of block $[F(4,280)=48.8, p<.001$, $\left.M S_{\mathrm{e}}=2,556\right]$ as RTs changed across trial blocks and a reliable interaction $\left[F(8,280)=6.3, p<.001, M S_{\mathrm{e}}=2,556\right]$. The critical change across blocks was from Block 4 to Block 5, when the random stimuli were introduced, which is the measure of sequence learning. A difference score from Block 5 and Block 4 may be used as a measure of learning, and these data are shown in the left part of Figure 5. An ANOVA of these learning scores shows no reliable differences among conditions $[F(2,70)=1.4, p>$ $\left..20, M S_{\mathrm{e}}=2,380\right]$. The difference score for the motor condition is not reliably different from zero $[t(27)=1.42$, $p>.20, S E M=9.8]$, but the difference scores for the perceptual and SRT conditions are (both $t \mathrm{~s}>3.0$ ). This unreliable difference in the training performance between the perceptual and motor conditions can only be attributed to random variation, as these two conditions were treated identically during training. It should be borne in mind that subjects in the motor and perceptual conditions are learning not only the sequence but also the incompatible mapping. Thus, the difference score from Block 4 to Block 5 may underestimate sequence learning; RTs may increase upon transfer to Block 5 due to the stimuli's becoming random (and thus sequence knowledge no longer providing an advantage), but RTs may continue to decrease as subjects continue to learn the incompatible stimulus-response mapping.

The transfer data are shown in Figure 4. There was not an overall effect of condition on RT $[F(2,70)=1.0, p>$ $\left..20, M S_{\mathrm{e}}=11,995\right]$, but there was a reliable difference in RTs across trial blocks $\left[F(2,140)=46.0, p<.001, M S_{\mathrm{e}}=\right.$ 455]. Most important, the difference across trial blocks varied with condition $\left[F(4,140)=2.8, p<.05, M S_{\mathrm{e}}=\right.$ 


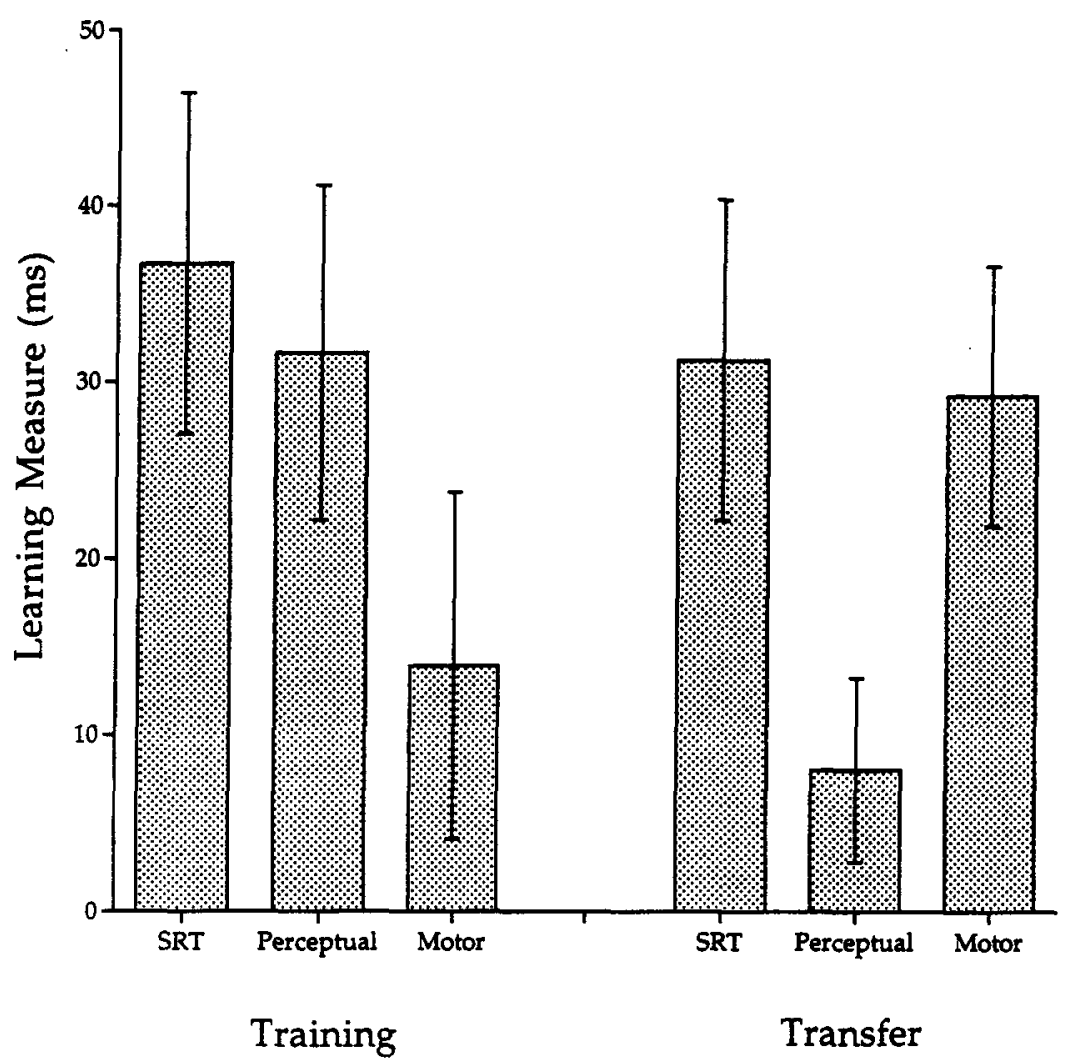

Figure 5. Summary learning measure across conditions at training (left) and transfer (right) for Experiment 3. Error bars are standard errors.

455]. This interaction was evaluated by comparing RTs on Blocks 7 (sequenced) and 8 (random); Block 6 RTs were not included in calculating the learning measure, because subjects in the motor and perceptual condition were still adjusting to the change in the stimulus-response mapping, as is clear, because Block 8 RTs msec are reliably faster than Block 6 RTs (average difference $=16 \mathrm{msec}$; both $t \mathrm{~s}>2.3$ ). In Experiments 1 and 2, RTs to the random blocks that were adjacent to the sequenced blocks were not reliably different from each other (average difference $=4 \mathrm{msec}$; all $t \mathrm{~s}<1.0$ ).

The learning measures are shown in the right part of Figure 5, and an ANOVA shows a reliable difference in the transfer scores $\left[F(2,70)=3.6, p<.05, M S_{\mathrm{e}}=1,192\right]$. Follow-up contrasts show that the perceptual condition showed less learning at transfer than did either of the other two conditions $(F \mathrm{~s}>4.8, p \mathrm{~s}<.05)$ and that the motor and SRT conditions did not differ $(F<1)$. The transfer shown by the perceptual condition differs from zero only marginally $[t(28)=1.5, .05<p<.10]$, and this marginal learning may have taken place during the single sequence block administered at transfer.

In looking at Figure 4, it might seem odd that the mean transfer RTs of the motor condition is not faster than the mean of the perceptual condition, inasmuch as the motor condition subjects are supposed to be showing knowledge of the sequence and the perceptual subjects are not. Although their overall RTs are approximately equal at transfer, the perceptual condition was marginally faster during training $(F=3.0, p=.08)$ and the perceptual condition loses that marginal RT advantage at transfer.

Experiment 3 supports the conclusion drawn from Experiment 2 that learning cannot be purely perceptual. It also makes clear that learning must have a motoric component.

\section{GENERAL DISCUSSION}

\section{What Is Learned?}

Prior work in this area has pointed towards the possibility that implicit perceptual-motor sequence learning can be supported by purely perceptual information. Two issues prevent a straightforward interpretation of these data. In some of the studies, subjects obtained substantial explicit knowledge of the sequence (Fendrich et al., 1991; Howard et al., 1992). Another concern is that watching spatial stimuli entails eye movements possibly bringing motoric artifacts into what is meant to be a perceptual experience (Mayr, 1996; Stadler, 1989).

In Experiment 1, subjects who watched but did not respond to stimuli showed some sequence knowledge, once asked to respond to the stimuli. This knowledge must have 
been largely explicit, because once the few subjects who could verbally report most of the sequence were removed from the analysis, the remaining subjects showed no benefit of having watched the sequence as compared with a group that had watched random stimuli. Experiment 2 ensured that subjects attended to the stimuli and removed the possibility that they could learn a sequence of eye movements by using centrally presented digits as stimuli. Subjects showed robust transfer of sequence knowledge when the stimuli were changed to spatial cues, which again indicates that the knowledge is not based on the stimuli. Experiment 3 showed that if the stimulus sequence is changed but the motor requirements remain the same, subjects express sequence knowledge, but that if the motor requirements change, subjects do not express sequence knowledge, even if the stimulus sequence is unchanged. Together, these three experiments show that implicit motor sequence learning is not purely perceptual.

The present results are consistent with the idea that sequence learning is motoric, but there is very strong evidence from other studies that learning is not purely motoric (A. Cohen et al., 1990; Keele et al., 1995). Given the power limitations of the present study (and others), it would be premature to conclude that perceptual and motor representations make no contribution to implicit learning, but it is quite clear that neither can support implicit learning alone, and the data also indicate that if they do contribute to learning, it is not a large part of the effect. What, then, are subjects learning?

Willingham et al. (1989) suggested that a sequence of stimulus-response action pairs are learned. The present results are not consistent with that proposal, however. In Experiment 2, robust transfer was observed when the stimuli were changed from digits to spatial cues. Changing the stimuli necessarily changes the stimulus-response pairs and therefore should have disrupted the expression of sequence knowledge. Furthermore, in Experiment 3 the mapping of stimuli and responses was changed, but the expression of sequence knowledge was not affected, as long as the sequence of responses remained the same. Thus, the learning seems to be neither purely perceptual, nor purely motoric, or a series of stimulus-response pairings.

Another possibility is that subjects learn a series of response locations (Willingham, 1998a). That is, they learn a series of locations in egocentric space to which they should respond; each location is a target for a movement. These targets are not tied to an effector; they are locations in space to which any effector can move. They are represented in egocentric space, meaning that they are coded relative to the person moving. Egocentric space may be contrasted with allocentric space, in which objects are coded in a spatial frame relative to one another. Thus, in the SRT task, the sequence of response locations would correspond to a sequence of locations of the keys subjects press to respond to the stimuli. Sequence learning in the SRT task is motoric in that it is coded in a spatial frame that is privileged to motor representations, but it is not motoric in the sense of being specific to an effector. Willingham (1998a) has suggested that this representation is used in all tasks that entail a repetitive sequence of movements.

This proposal changes the account of some of the studies arguing for perceptual learning. Both Stadler (1989) and Mayr (1996) reported perceptual learning but admitted that subjects may have been learning a sequence of eye movements. Under the current proposal, subjects were not learning a sequence of movements per se but rather a sequence of spatial targets to which they should move their eyes (or move attention).

There is good evidence from single-cell recording studies in nonhuman primates that target selection is coded in egocentric space in primary and secondary motor cortices (e.g., Graziano, Yap, \& Gross, 1994) and also good evidence that these cortical areas are crucial to motor-skill learning (Grafton et al., 1992; Grafton, Woods, \& Tyszka, 1994). Thus, it is at least plausible that implicit sequence learning is represented as a sequence of egocentric response locations. There has not been a direct test of this proposal, however, and the best evidence supporting it is that there are data weighing against the other plausible alternatives.

\section{The Specificity of Implicit Learning}

A number of researchers have suggested that learning in implicit tasks differs fundamentally from learning in explicit tasks in that the former is inflexible or shows hyperspecificity (Berry \& Dienes, 1993; N. J. Cohen, Poldrack, \& Eichenbaum, 1997; Dienes \& Berry, 1997; Reber, Knowlton, \& Squire, 1996; Squire, 1992, 1994). They propose that explicit knowledge may be applied in contexts quite different from those in which it was encoded, whereas implicit knowledge can be applied only in situations quite similar to those in which it was encoded. The data reported here show that implicit learning can actually be quite flexible.

Although I argued in another paper for the apparent specificity of SRT learning (Willingham et al., 1989), I have since argued that this characterization is an oversimplification (Willingham, 1997, 1998b; Willingham \& Preuss, 1995). Although the data on specificity is compelling in the animal literature (e.g., Eichenbaum, Fagan, Mathews, \& Cohen, 1989), there is a conceptual problem with this work in human subjects. One argument for the specificity of learning on implicit tasks is that changes in the stimulus characteristics seem to eliminate the learning. For example, changing the modality of presentation or the type font of words drastically reduces the level of repetition priming, whereas performance on explicit measures, such as recognition and recall, are resistant to such changes (e.g., Graf, Shimamura, \& Squire, 1985). But repetition priming depends on the structural characteristics of stimuli, whereas recognition and recall 
depend on the semantic content of the stimuli; it is not shocking that changing the structural characteristics affects implicit, but not explicit, measures.

Data from the present experiments provide an example of the flexibility of implicit memory. In Experiment 2, the stimuli were changed from digits to spatial cues, and learning transferred. In Experiment 3 the stimulus-response mapping was changed and learning transferred (as long as the motor sequence was unchanged). These results are consistent with the idea that the apparent specificity or generality of learning depends not on whether the learning is implicit or explicit, but on whether the characteristic that is changed is crucial to the representation of learning. (For a fuller treatment of this topic, see Willingham, 1997, 1998b).

\section{Implications for Neural Accounts of Implicit Learning}

Data from imaging studies and from neurological patients support the interpretation that subjects learn sequences of responses rather than sequences of stimuli. Studies using positron emission tomography (Grafton, Hazeltine, \& Ivry, 1995) and transcranial magnetic stimulation (Pascual-Leone, Grafman, \& Hallett, 1994) indicate that the neural structures underlying motor-skill learning include the basal ganglia and the secondary and primary motor cortex. Supporting that proposal is the finding that patients who have been reported to fail to learn the SRT task are those with motor disorders. Patients with striatal abnormalities like Huntington's disease (HD) fail to learn the sequence (Knopman \& Nissen, 1991; Willingham \& Koroshetz, 1993), as do patients with Parkinson's disease (PD) (Jackson, Jackson, Harrison, Henderson, \& Kennard, 1995; but see Pascual-Leone et al., 1993) and patients with cerebellar damage (PascualLeone et al., 1993). The results reported here support the idea that learning occurs through plasticity in the same brain regions that support motor control.

One might question this conclusion by proposing that patients do not fail to learn because of damage to motor structures. Rather, it may be that the integrity of the frontal lobe is necessary to normal sequence learning. Caudate degeneration associated with HD may deafferent the frontal lobe, and PD also leads to degeneration of the ventral tegmental area (Uhl, Hedreen, \& Price, 1985), which would deprive the frontal lobe of dopamine. Furthermore, there are substantial projections from the frontal lobe to the cerebellum, and it has been implicated in cognitive tasks (e.g., Kim, Ugurbil, \& Strick, 1994). The imaging data might be accounted for by proposing that the activation observed in motor areas does not reflect learning but derives from other structures that are actually doing the learning. Recent evidence, however, indicates that patients with frontal lobe damage are able to learn the SRT task normally (Marks \& Cermak, 1996). Thus, it appears unlikely that the apparent involvement of motor structures in SRT learning is actually secondary to frontal involvement.
Thus, the present data set indirectly support proposals that motor-skill learning occurs in the same neural structures that support motor control (Grafton et al., 1992; Kawashima, Roland, \& O'Sullivan, 1995; Willingham, 1992, 1998a).

\section{REFERENCES}

BERRY, D. C., \& DiENES, Z. (1993). Implicit learning: Theoretical and empirical issues. Hillsdale, NJ: Erlbaum.

COHEN, A., IVRY, R. I., \& KeELE, S. W. (1990). Attention and structure in sequence learning. Journal of Experimental Psychology: Learning, Memory, \& Cognition, 16, 17-30.

Cohen, N. J., Poldrack, R. A., \& Eichenbaum, H. (1997). Memory for items and memory for relations in the procedural/declarative memory framework. Memory, 5, 131-178.

Curran, T., \& Kefle, S. W. (1993). Attentional and nonattentional forms of sequence learning. Journal of Experimental Psychology: Learning, Memory, \& Cognition, 19, 188-202.

DIENES, Z., \& BERRY, D. (1997). Implicit learning: Below the subjective threshold. Psychonomic Bulletin \& Review, 4, 3-23.

Eichenbaum, H., Fagan, A., Mathews, P., \& Cohen, N. J. (1989). Further studies of hippocampal representation during odor discrimination learning. Behavioral Neuroscience, 103, 1207-1216.

Fendrich, D. W., Healy, A. F., \& Bourne, L. E., JR. (1991). Longterm repetition effects for motoric and perceptual procedures. Journal of Experimental Psychology: Learning, Memory, \& Cognition, 17, 137-151.

Graf, P., Shimamura, A. P., \& Squire, L. R. (1985). Priming across modalities and priming across category levels: Extending the domain of preserved function in amnesia. Journal of Experimental Psychology: Learning, Memory, \& Cognition, 10, 386-396.

Grafton, S. T., Hazeltine, E., \& IVRY, R. (1995). Localization of independent cortical systems in human motor learning. Journal of Cognitive Neuroscience, $7,497-510$.

Grafton, S. T., Mazziotta, J. C., Presty, S., Friston, K. J., Frackowiak, R. S. J., \& PhelPS, M. E. (1992). Functional anatomy of human procedural learning determined with regional cerebral blood flow and PET. Journal of Neurosciences, 12, 2542-2548.

Grafton, S. T., Woods, R. P., \& TYszKa, M. (1994). Functional imaging of procedural motor learning: Relating cerebral blood flow with individual subject performance. Human Brain Mapping, 1, 221-234.

Graziano, M. S. A., YaP, G. S., \& Gross, C. G. (1994). Coding of visual space by premotor neurons. Science, 266, 1054-1057.

Howard, J. H., MutTer, S. A., \& Howard, D. V. (1992). Serial pattern learning by event observation. Journal of Experimental Psychology: Learning, Memory, \& Cognition, 18, 1029-1039.

JaCKSON, G. M., JACKSON, S. R., HARRISON, J., Henderson, L., \& KENNARD, C. (1995). Serial reaction time learning and Parkinson's disease: Evidence for a procedural learning deficit. Neuropsychologia, 33, 577-593

Kawashima, R., Roland, P. E., \& O'Sullivan, B. T. (1995). Functional anatomy of reaching visuomotor learning: A positron emission tomography study. Cerebral Cortex, 2, 111-122.

Keele, S. W., Jennings, P., Jones, S., Caulton, S., Caulton, D., \& COHEN, A. (1995). On the modularity of sequence representation. Journal of Motor Behavior, 27, 17-30.

KIM, S., UgURBIL, K., \& STRICK, P. L. (1994). Activation of a cerebellar output nucleus during cognitive processing. Science, 265, 949-951.

Knopman, D., \& Nissen, M. J. (1991). Procedural learning is impaired in Huntington's disease: Evidence from the serial reaction time task. Neuropsychologia, 29, 245-254.

MARKS, A. R., \& CERMAK, L. S. (1996). Etiological differences in the amnesic syndrome: Performance of amnesic patients and patients with frontal-lobe damage on the serial reaction time task. Manuscript submitted for publication.

MAYR, U. (1996). Spatial attention and implicit sequence learning: Evidence for independent learning of spatial and nonspatial sequences. Journal of Experimental Psychology: Learning, Memory, \& Cognition, 22, 350-364 
Nissen, M. J., Willingham, D. B., \& Hartman, M. (1989). Explicit and implicit remembering: When is learning preserved in amnesia? Neuropsychologia, 27, 341-352.

Pascual-Leone, A., Grafman, J., Clark, K., Stewart, M., MasSAquOI, S., Lou, J.-S., \& HALleTt, M. (1993). Procedural learning in Parkinson's disease and cerebellar degeneration. Annals of Neurology, 34, 594-602.

Pascual-Leone, A., Grafman, J., \& Hallett, M. (1994). Modulation of cortical motor output maps during development of implicit and explicit knowledge. Science, 263, 1287-1289.

Reber, P. J., Knowlton, B. J., \& SQuire, L. R. (1996). Dissociable properties of memory systems: Differences in the flexibility of declarative and nondeclarative knowledge. Behavioral Neuroscience, $110,861-871$

REBER, P. J., \& Squire, L. R. (1994). Parallel brain systems for learning with and without awareness. Learning \& Memory, 1, 217-229.

SQuire, L. R. (1992). Memory and the hippocampus: A synthesis from findings with rats, monkeys, and humans. Psychological Review, 99, 195-231.

SQuiRE, L. R. (1994). Declarative and nondeclarative memory: Multiple brain systems supporting learning and memory. In D. Schacter \& E. Tulving (Eds.), Memory Systems 1994 (pp. 203-231). Cambridge, MA: MIT Press.

STADLER, M. A. (1989). On learning complex procedural knowledge. Journal of Experimental Psychology: Learning, Memory, \& Cognition, 15, 1061-1069.

Uhl, G. R., Hedreen, J. C., PrJce, D. L. (1985). Parkinson's disease: Loss of neurons from the ventral tegmental area contralateral to therapeutic surgical lesions. Neurology, 35, 1215-1218.

WeLFORD, A. T. (1976). Skilled performance: Perceptual and motor skills. Glenview, IL: Scott, Foresman.
Willingham, D. B. (1992). Systems of motor skill. In L. R. Squire \& N. Butters (Eds.), Neuropsychology of Memory (2nd ed., pp. 166178). New York: Guilford.

WiLlinghaM, D. B. (1997) Implicit and explicit memory do not differ in flexibility: Comment on Dienes and Berry (1997). Psychonomic Bulletin \& Review, 4, 587-591.

Willingham, D. B. (1998a). COBALT: A neuropsychological theory of motor skill learning. Psychological Review, 105, 558-584.

WiLlingham, D. B. (1998b). What differentiates declarative and procedural memories: Reply to Cohen, Poldrack, and Eichenbaum (1997). Memory, 6, 689-699.

Willingham, D. B., Greeley, T., \& Bardone, A. (1993). Dissociation in a serial response time task using a recognition measure: Reply to Perruchet and Amorim. Journal of Experimental Psychology: Learning, Memory, \& Cognition, 19, 1424-1430.

Willingham, D. B., \& Koroshetz, W. J. (1993). Evidence for dissociable motor skills in Huntington's disease patients. Psychobiology, 21, 173-182.

Willingham, D. B., Nissen, M. J., \& Bullemer, P. (1989). On the development of procedural knowledge. Journal of Experimental Psychology: Learning. Memory, \& Cognition, 15, 1047-1060.

Willingham, D. B., \& Preuss, L. (1995). The death of implicit memory. Available: PSYCHE, 2. http://psyche.cs.monash.edu.au/volume 2-1/ psyche-95-2-15-implicit-1-willingham.html

ZIEßLER, M. (1994) The impact of motor responses on serial-pattern learning. Psychological Research, 57, 30-41.

(Manuscript received February 20, 1997; accepted for publication September 17, 1997.) 